\title{
Effects of Arkadia on airway remodeling through enhancing TGF- $\beta$ signaling in allergic rats
}

\author{
Xiao-Zhao $\mathrm{Li}^{1,2,4}$, Jun-Tao Feng ${ }^{1,4}$, Cheng-Ping Hu${ }^{1}$, Ze-Qi Chen ${ }^{3}$, Qi-Hua Gu ${ }^{1}$ and Hua-Ping Nie
}

Upregulation of transforming growth factor- $\beta$ (TGF- $\beta$ ) signaling is interrelated with the development of airway remodeling. In this study, we examined the role of two E3 ubiquitin ligases, Arkadia and Smurf2, which are critically required for TGF- $\beta$ signaling in airway remodeling. Rats were immunized with ovalbumin (OVA) and then challenged with an OVA aerosol. In in vitro experiments, normal human bronchial epithelial cells were stimulated with TGF- $\beta_{1}$ with or without the preincubation of Arkadia/Smurf2 small interfering RNA (siRNA) or lactacystin (an inhibitor of proteasomal degradation). In the lungs of OVA-treated rats, a large number of inflammatory cells were present near the airways. An increased subepithelial collagen deposition was associated with high expression levels of Smad7, SnoN and Ski mRNAs, Arkadia, Smurf2, and TGF- $\beta$ type I receptor (T $\beta$ RI), but low expression levels of Smad7, SnoN and Ski proteins. Smad7, SnoN and Ski interacted with both Arkadia and Smurf2 while T $\beta$ RI only interacted with Smurf2 but not with Arkadia. In in vitro experiments, the inhibitory effect of TGF- $\beta_{1}$ on the expression of Smad7, SnoN and Ski was reversed by Arkadia siRNA and lactacystin, whereas the stimulatory effect of TGF- $\beta_{1}$ on the expression of T $\beta$ RI protein and Smad7/SnoN/Ski mRNAs was not affected. In contrast, Smurf2 siRNA did not influence the effects of TGF- $\beta_{1}$ on the expression of the above proteins. Our results suggest that Arkadia may contribute to the pathogenesis of airway remodeling through enhancing TGF- $\beta$ signaling by inducing the reduction of Smad7, SnoN and Ski proteins in OVA-sensitized and -challenged rats. Laboratory Investigation (2010) 90, 997-1003; doi:10.1038/labinvest.2010.78; published online 12 April 2010

KEYWORDS: airway remodeling; Arkadia; Ski; Smad7; SnoN; TGF- $\beta$ signaling

Asthma is a chronic inflammatory disease of the airways, and recurrent inflammation results in airway remodeling characterized by subepithelial fibrosis, myofibroblast and myocyte hyperplasia, together with epithelial damage, goblet cell metaplasia, edema and increased vascularity. ${ }^{1}$ Transforming growth factor $\beta$ (TGF- $\beta$ ), a fibrogenic cytokine, has been implicated in the development of airway remodeling in asthma on the basis of its strong capacity to induce the production of extracellular matrix factors including type 1 collagen (COL-I). ${ }^{2}$ It is, therefore, possible that regulators of TGF- $\beta$ activity might also have important role in the modulation of the airway remodeling process in asthma.

The process of TGF- $\beta$ signaling pathway activation is known as follows. On TGF- $\beta$ stimulation and activation of the TGF- $\beta$ type I receptor (T $\beta \mathrm{RI}$ ), R-Smads (Smad2 and Smad3) are phosphorylated. Then, phosphorylated R-Smads bind to Smad4, and are translocated into the nucleus, in which such a complex, together with additional DNA-binding co-factors, controls the transcription of TGF- $\beta$ responsive genes. ${ }^{3}$ In the process, negative regulators such as Smad7, SnoN and Ski can downregulate TGF- $\beta$ signaling through multiple mechanisms. ${ }^{3,4}$ Accumulative evidence indicates that the expression of Smad7, SnoN and Ski proteins in some fibrotic models is obviously lower than that in the normal controls, ${ }^{5-13}$ and also shows that upregulation of the expression of Smad7, SnoN or Ski proteins reverses or blocks fibrogenesis. $^{14-17}$ Nakao et al ${ }^{18}$ reported that Smad7 immunoreactivity, which was present mainly in bronchial epithelial cells, was markedly decreased in asthmatic patients, compared with that of control subjects. However, the expression levels of SnoN and Ski in asthmatic patients are unclear.

\footnotetext{
${ }^{1}$ Department of Respiratory Medicine, Xiangya Hospital, Central South University, Changsha, China; ${ }^{2}$ Postdoctoral Station of Integrated Traditional and Western Medicine, Xiangya Hospital, Central South University, Changsha, China and ${ }^{3}$ Institute of Integrated Traditional Chinese and Western Medicine, Xiangya Hospital, Central South University, Changsha, China

Correspondence: Professor C-P Hu, MD, Department of Respiratory Medicine, Xiangya Hospital, Central South University, Xiangya Road No. 87, Changsha, Hunan, 410008, China. E-mail: huchengp28@yahoo.com.cn

${ }^{4}$ These authors contributed equally to this work.

Received 12 October 2009; revised 9 February 2010; accepted 22 February 2010
} 
The expression of Smad7, SnoN and Ski is modulated by the ubiquitin-proteasome degradation pathway, ${ }^{19-21}$ and in the process of regulation, E3 ubiquitin ligases, such as Arkadia and Smurf2, induce ubiquitin-dependent degradation of Smad7, SnoN and Ski. ${ }^{11-13,19-23}$ Whereas Fukasawa et $a l^{6,13}$ reported that Smurf2 mediated the degradation of Smad7, SnoN and Ski in a mouse model of renal tubulointersitial fibrosis, Liu $e t a l^{7,24}$ have shown that the reduction of Smad7 in a rat model of renal tubulointersitial fibrosis and normal human renal tubular epithelial cells may be mainly attributed to Arkadia, but not to Smurf2. Thus, how Arkadia and Smurf2 affect Smad7, SnoN and Ski in organ fibrosis remains to be clarified. Moreover, the interactions between Arkadia/Smurf2 and Smad7/SnoN/Ski in allergic airway remodeling are unknown. Therefore, this study was designed to clarify the effects of Arkadia and Smurf2 on the expression of Smad7, SnoN, Ski as well as T $\beta$ RI in pathogenesis of airway remodeling in ovalbumin (OVA)-sensitized and -challenged rats in vivo and TGF- $\beta_{1}$-treated bronchial epithelial cells in vitro.

\section{MATERIALS AND METHODS} Animals

All animals used in this study were 6- to 8-week-old male Sprague-Dawley rats obtained from the Experimental Animal Center of Central South University (Changsha, China). Animal study protocols were approved by the Animal Care Committee of Central South University and the experiments were carried out according to the guidelines of the committee.

\section{Chronic Airway Remodeling Model}

Eight rats were sensitized and challenged with OVA (OVAtreated group) to induce chronic airway remodeling. Briefly, the rats were immunized with an intraperitoneal injection of $100 \mathrm{mg}$ of OVA (Sigma, St Louis, MO, USA), $200 \mathrm{mg}$ of aluminum hydroxide (Sigma), and $6 \times 10^{9}$ heat-killed Bordetella pertussis (Wuhan Institute of Biological Products, Wuhan, China) in $1 \mathrm{ml}$ normal saline on days 0 and 14. From day 21 , the rats were challenged for $30 \mathrm{~min}$ by nebulization of $1 \%$ (wt/vol) OVA in saline solution on 3 day/week for 6 weeks. As a control group, eight rats were sensitized with $1 \mathrm{ml}$ normal saline and challenged with nebulized saline solution. All animals were killed $48 \mathrm{~h}$ after the final antigen or saline challenge.

\section{Cell Culture and Treatment}

Normal human bronchial epithelial cells (NHBECs) (Cell Center of Central South University, Changsha, China) were grown in a $5 \% \mathrm{CO}_{2}$ atmosphere at $37^{\circ} \mathrm{C}$ in Dulbecco's modified Eagle's medium containing 10\% fetal bovine serum in six-well plastic plates for $24 \mathrm{~h}$, and then a recombinant human TGF- $\beta_{1}$ (R\&D Systems, Minneapolis, MN, USA) at a final concentration of $5 \mathrm{ng} / \mathrm{ml}$ was added into the cell culture. The cells were collected $72 \mathrm{~h}$ later, and the expression of
Arkadia, Smurf2, T $\beta$ RI, Smad7, SnoN and Ski proteins and mRNAs in TGF- $\beta_{1}$-stimulated NHBECs were detected by western blot and real-time RT-PCR, respectively. In an additional experiment, NHBECs were cultured with or without Arkadia, Smurf2 or control small interfering RNA (siRNA) ( $2 \mu \mathrm{g} / \mathrm{ml}$, Sofarbio Technology, Hangzhou, China) for $24 \mathrm{~h}$ or lactacystin $\left(10 \mu \mathrm{M}\right.$, Sigma) for $3 \mathrm{~h}$. Then, TGF- $\beta_{1}$ was added into the cell culture as described above. The cells were collected $72 \mathrm{~h}$ later, and the expression of Arkadia, Smurf2, T $\beta$ RI, Smad7, SnoN and Ski proteins and mRNAs in TGF$\beta_{1}$-stimulated NHBECs were detected as described above.

\section{Histopathological Staining}

Lung tissues were fixed in $4 \%$ paraformaldehyde in phosphate-buffered saline and embedded in paraffin. Tissue sections ( $4 \mu \mathrm{m}$ thick) were stained with hematoxylin and eosin and Masson's trichrome for histopathological examination.

\section{Western Blotting}

The lung tissues and NHBECs were lysed with Nonidet P-40 (NP-40) lysis buffer as previously described. ${ }^{7,24}$ Proteins $(20 \mu \mathrm{g})$ were loaded and separated by SDS-polyacrylamide gel electrophoresis (SDS-PAGE) and transferred to PolyScreen polyvinylidine difluoride transfer membranes (Pharmacia Corporation, Piscataway, NJ, USA). Immunoblotting was performed with different antibodies and visualized by the enhanced chemiluminescence (Amersham, Piscataway, NJ, USA) method. The primary antibodies purchased from Santa Cruz Biotechnology (Santa Cruz, CA, USA) were goat anti-Arkadia, goat anti-Smurf2, goat anti-SnoN, rabbit anti-Smad7, rabbit anti-Ski and rabbit anti-T $\beta$ RI. Mouse monoclonal anti- $\beta$-actin (Sigma) was used as an internal control.

\section{Immunoprecipitation}

Each extract from lung tissues was incubated with $4 \mu \mathrm{g}$ of specific anti-Smad7, anti-SnoN, anti-Ski or anti-T $\beta$ RI antibody and $50 \mu$ lof protein G-Sephrose beads for $3 \mathrm{~h}$ at $4{ }^{\circ} \mathrm{C}$. The immunoprecipitates were washed three times with cold lysis buffer and then separated by SDS-PAGE for immunoblotting of associated proteins in the complex. The lysates incubated with anti-rabbit IgG or anti-goat IgG, instead of the specific antibodies were used as negative controls.

\section{Real-Time RT-PCR}

Total RNA was extracted from the lung tissues and NHBECs by using Trizol (Invitrogen, Carlsbad, CA, USA). Reverse transcription of the RNA was performed by using a first-strand complementary DNA synthesis kit (Promega, Madison, MI, USA). The PCR with SYBR Green PCR Master Mix (Takara, Shiga, Japan) was used to analyze the mRNA expression of TGF- $\beta_{1}$, COL-I, Smad7, SnoN and Ski. Realtime PCR was performed by using the ABI PRISM 7900-HT Sequence Detection System (Applied Biosystems, Foster City, CA, USA). The primer sequences used were as follows: rat TGF- $\beta_{1}$ : forward $5^{\prime}$-CCTGAGTGGCTGTCTTTTGACG-3', 
reverse $5^{\prime}$-AGTGAGCGCTGAATCGAAAGC- ${ }^{\prime}$; rat COL-I: forward $5^{\prime}$-AGAGCATGACCGATGGATTCC- $3^{\prime}$, reverse $5^{\prime}$ TTGCCAGTCTGCTGGTCCATG-3'; rat Smad7: forward 5'TCGGACAGCTCAATTCGGAC- ${ }^{\prime}$, reverse $5^{\prime}$-GGTAACTGC TGCGGTTGTAA-3'; rat SnoN: forward $5^{\prime}$-GAGGAGCAG GAGAAAATGG- ${ }^{\prime}$, reverse $5^{\prime}$-GAATCACGCTTGCTTGTG$3^{\prime}$; rat Ski: forward 5'-TGACTCTGGACACAGCAGGA-3', reverse $5^{\prime}$-GAGAGGACAGCGAGGACAAG- $3^{\prime}$; rat $\beta$-actin: forward $5^{\prime}$-TGGGTCAGAAGGACTCCTATG- $3^{\prime}$, reverse: $5^{\prime}$ CAGGCAGCTCATAGCTCTTCT- $3^{\prime}$; human Smad7: forward 5'-CCTTAGCCGACTCTGCGAACTA-3' ${ }^{\prime}$, reverse $5^{\prime}$-CCAGAT AATTCGTTCCCCCTGT-3'; human SnoN: forward $5^{\prime}$-AAG GCTCACAGTAGTGGTAA- $3^{\prime}$, reverse $5^{\prime}$-GTGGCAGACTCA GATTTCC-3'; human Ski: forward 5'-CTGCTGAGCCAGG ATT- ${ }^{\prime}$, reverse $5^{\prime}$-TTGTACTTGTTGCCATAGTC-3'; and human $\beta$-actin: forward $5^{\prime}$-TGGGTCAGAAGGACTCCT ATG-3' ${ }^{\prime}$, reverse $5^{\prime}$-CAGGCAGCTCATAGCTCTTCT-3'. Realtime PCR data were analyzed according to the manufacturer's instructions.

\section{Statistical Analysis}

Data were presented as means \pm s.d. The differences in the expression levels of proteins and mRNAs between control rats and OVA-treated rats were determined by the independent samples $t$-test. The differences in the expression levels of proteins and mRNAs among control NHBECs and NHBECs treated with TGF- $\beta_{1}$, Arkadia siRNA, Smurf2 siRNA, control siRNA and/or lactacystin were determined by one-way ANOVA with least significant difference as an adjusted post hoc test. All statistical analyses were performed using SPSS 11.0 (SPSS, Chicago, IL, USA). A $P$-value of $<0.05$ was considered statistically significant.

\section{RESULTS \\ Histopathological Changes and the Expression of TGF- $\beta_{1}$ and COL-I in the Lungs of OVA-Treated Rats}

A large numbers of inflammatory cells were present near the airways and there was a significant increase in the subepithelial collagen deposition in the lungs of OVA-treated rats, compared with those of control rats (Figure 1a). These pathological changes were associated with a significant increase in TGF- $\beta_{1}$ and COL-I mRNAs expression in the lungs of OVA-treated rats, compared with those of control rats (Figure 1b).

\section{Reduction of Smad7, SnoN and Ski Proteins, but not mRNAs, in the Lungs of OVA-Treated Rats}

Western blotting showed that the expression of Smad7, SnoN and Ski proteins was significantly decreased in OVA-treated lungs, compared with saline-treated lungs (Figure 2a). To analyze whether the reduction of Smad7, SnoN and Ski proteins resulting from the downregulation of Smad7, SnoN and Ski mRNAs, we analyzed the mRNA expression of Smad7, SnoN and Ski by real-time RT-PCR. In contrast to the significant decrease of the three proteins, the expression of Smad7, SnoN and Ski mRNAs was increased in OVA-treated lungs, compared with saline-treated lungs (Figure 2b).

\section{Increased Expression of Arkadia, Smurf 2 and $T \beta R I$ in the Lungs of OVA-Treated Rats}

Western blotting showed that the expression of Arkadia, Smurf2 and T $\beta$ RI was significantly increased in OVA-treated lungs, compared with saline-treated lungs (Figure 3). a
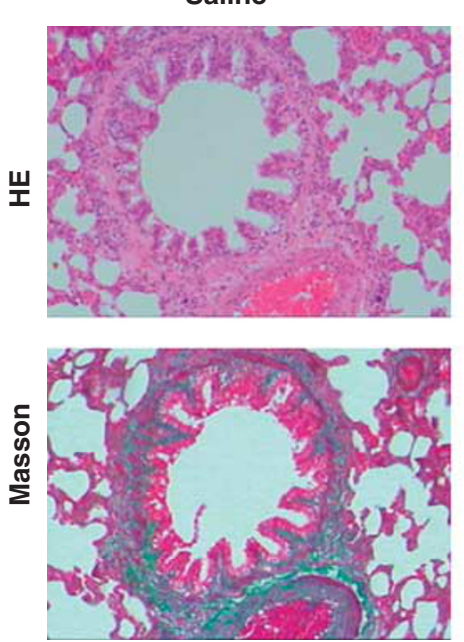

OVA
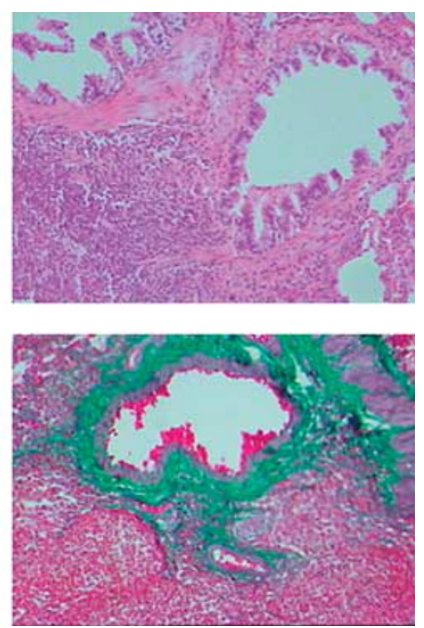

b

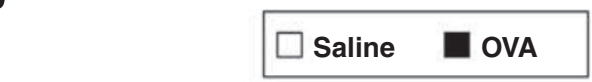

Figure 1 Histopathological changes and expression of TGF- $\beta_{1}$ and COL-1 mRNAs in the lungs of allergic rats. (a) H\&E staining and Masson's trichrome staining in OVA-treated (OVA) and saline-treated (saline) lungs (original magnification $\times 200$ ). Note: the progressive development of inflammatory infiltration and collagen deposition in OVA-treated lungs. (b) The relative ratios of TGF- $\beta_{1}$ and COL- 1 mRNAs to $\beta$-actin mRNA in OVA-treated lungs and saline-treated lungs. Real-time RT-PCR shows a significant increase of TGF- $\beta_{1}$ and COL- 1 mRNAs in the lungs of OVA-treated rats, compared with those of saline-treated rats. Data are expressed as mean \pm s.d. $(n=8)$. ${ }^{\star} P<0.01$, compared with saline-treated rats. 

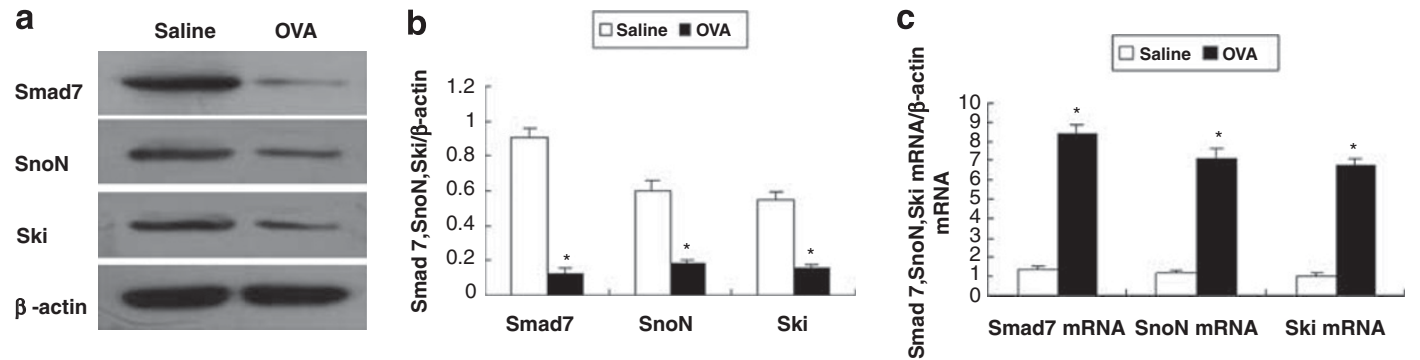

Figure 2 Expression levels of Smad7, SnoN, and Ski proteins and mRNAs in the lungs of allergic rats. (a) Western blotting shows a significant reduction in the expression of Smad7, SnoN and Ski proteins in OVA-treated lungs, compared with saline-treated lungs. Data are relative ratios of expression intensities of Smad7, SnoN and Ski to $\beta$-actin in OVA-treated lungs and saline-treated lungs. (b) The relative ratios of Smad7, SnoN and Ski mRNAs to $\beta$-actin mRNA in OVA-treated and saline-treated lungs. Real-time RT-PCR shows a significant increase in the expression of Smad7, SnoN and Ski mRNAs in OVA-treated lungs, compared with saline-treated lungs. Data are expressed as mean \pm s.d. $(n=8) .{ }^{*} P<0.01$, compared with saline-treated lungs.
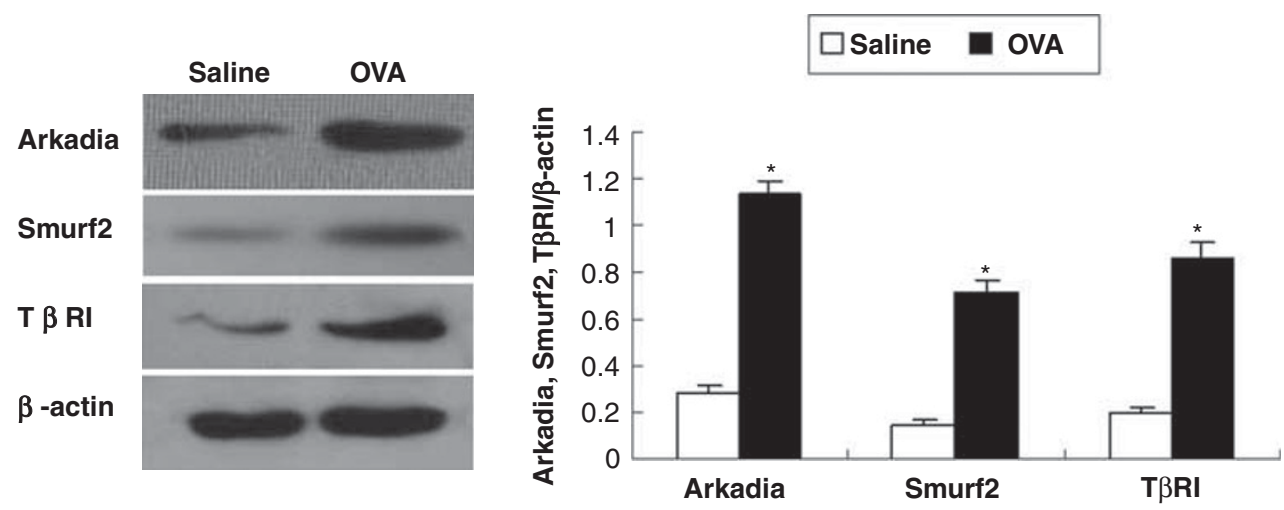

Figure 3 Expression levels of Arkadia, Smurf2 and T $\beta \mathrm{RI}$ in the lungs of allergic rats. Western blotting shows that the expression of Arkadia, Smurf2 and T $\beta \mathrm{RI}$ is increased in OVA-treated and saline-treated lungs. Data are relative ratios of expression intensities of Arkadia, Smurf2 and T $\beta$ RI to $\beta$-actin in OVA-treated lungs and saline-treated lungs. Data are expressed as mean \pm s.d. $(n=8) .{ }^{*}<0.01$, compared with saline-treated lungs.

\section{The Interactions of Arkadia/Smurf2 with Smad7/SnoN/ Ski/T/RI in the Lungs of OVA-Treated Rats}

To determine the effects of Arkadia and Smurf2 on TGF- $\beta$ signaling and airway remodeling, we analyzed the interactions of Arkadia/Smurf2 with Smad7, SnoN, Ski and T $\beta$ RI in the lungs of OVA-treated rats using co-immunoprecipitation. In OVA-treated lungs, Arkadia-Smad7, Smurf2-Smad7, Arkadia-SnoN, Smurf2-SnoN, Arkadia-Ski and Smurf2-Ski complexes were all observed (Figures $4 \mathrm{a}-\mathrm{c}$ ). In addition, the Smurf2-T $\beta$ RI complex was observed; however, there was no complex composed of Arkadia and T $\beta$ RI (Figure $4 \mathrm{~d}$ ). These findings indicate that both Smurf2 and Arkadia interact with Smad7, SnoN and Ski, respectively, and Smurf2, but not Arkadia, interacts with T $\beta$ RI in OVA-treated lungs.

\section{Effects of Preincubation of Arkadia siRNA on the Expression of Arkadia, T/RI, Smad7, SnoN and Ski in TGF- $\beta_{1}$-Stimulated NHBECs}

Western blotting showed that the expression of Arkadia and T $\beta$ RI were increased, while that of Smad7, SnoN and Ski were decreased in TGF- $\beta_{1}$-stimulated NHBECs, compared with control NHBECs, The expression of Arkadia in TGF- $\beta_{1^{-}}$ stimulated NHBECs was inhibited by preincubation of Ar- kadia siRNA or lactacystin, while control siRNA did not affect the expression of Arkadia. Similar to the control siRNA, neither Arkadia siRNA nor lactacystin affected the expression of T $\beta$ RI in TGF- $\beta_{1}$-stimulated NHBECs. The low expression of Smad7, SnoN and Ski in TGF- $\beta_{1^{-}}$ stimulated NHBECs was reversed by Arkadia siRNA or lactacystin, but not by the control siRNA (Figures 5a-c). However, real-time RT-PCR showed that Arkadia siRNA or lactacystin did not significantly affect the mRNA expression of Smad7, SnoN and Ski in TGF- $\beta_{1}$-stimulated NHBECs (Figure 5d).

\section{Effects of Preincubation of Smurf2 siRNA on the Expression of Smurf2, T/RI, Smad7, SnoN and Ski in TGF- $\beta_{1}$-Stimulated NHBECs}

Similarly, western blotting showed that the expression of Smurf2 and TRRI was increased, while Smad7, SnoN and Ski were decreased in NHBECs stimulated with TGF- $\beta_{1}$, compared with control NHBECs. In addition, the expression of Smurf2 in TGF- $\beta_{1}$-stimulated NHBECs was inhibited by preincubation of Smurf2 siRNA or lactacystin, but not by the control siRNA. Similar to the control siRNA and lactacystin, preincubation of Smurf2 siRNA did not affect the expression 
a

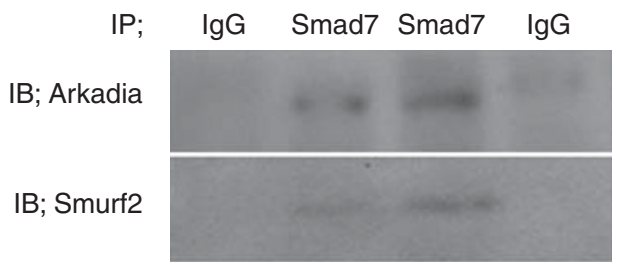

C

(P);

IB; Arkadia

IB; Smurf2

Saline

Saline

OVA

;

IgG Ski Ski IgG
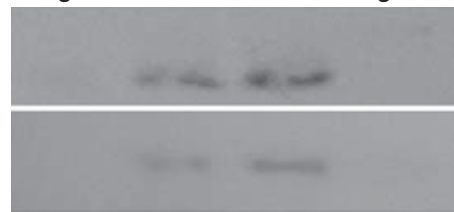

b

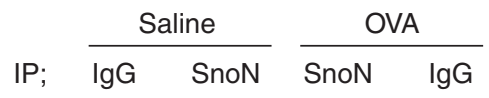

IB; Arkadia

IB; Smurf2

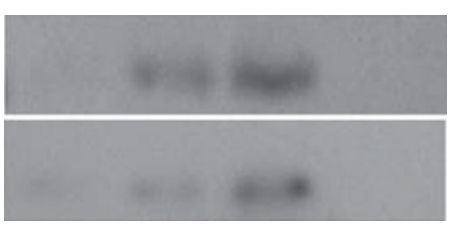

d

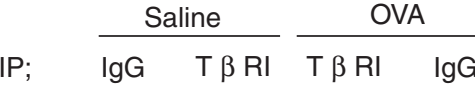

IB; Arkadia

IB; Smurf2

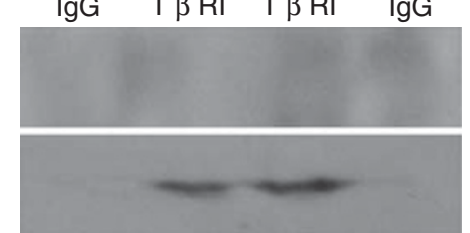

Figure 4 The interactions of Arkadia/Smurf2 with Smad7/SnoN/Ski/T $\beta R \mathrm{I}$ in the lungs of allergic rats. Lung extracts collected from OVA-treated and salinetreated rats were subjected to Smad7, SnoN, Ski or T $\beta$ RI immunoprecipitation (IP), followed by Arkadia or Smurf2 immunoblotting (IB). In OVA-treated lungs, Smad7-Arkadia and Smad7-Smurf2 (a), SnoN-Arkadia and SnoN-Smurf2 (b), Ski-Arkadia and Ski-Smurf2 (c) and T $\beta$ RI-Smurf2 (d) complexes are noted. However, there is no T $\beta$ RI and Arkadia complex. Representative data of three independent experiments are shown.

a

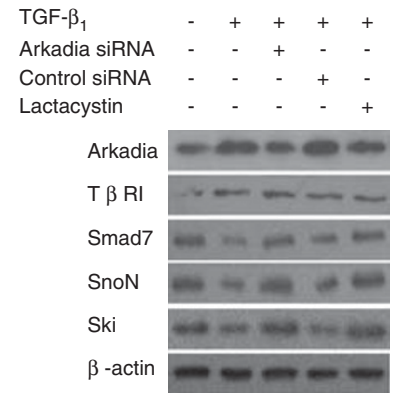

b

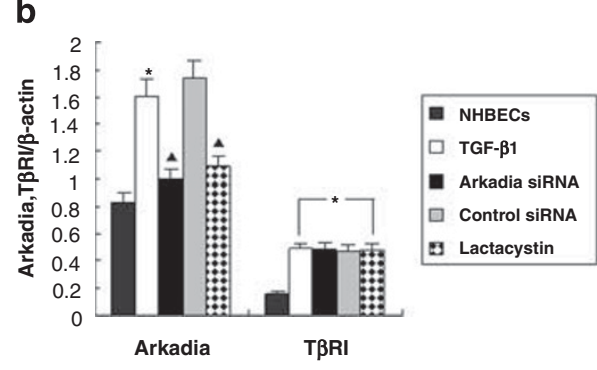

C

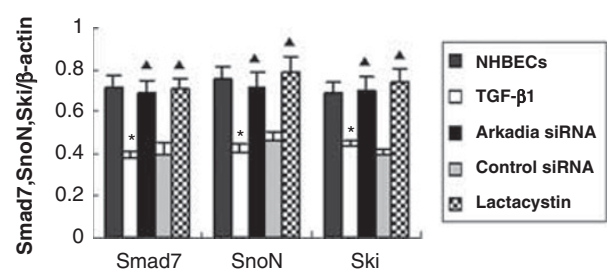

Figure 5 Effects of Arkadia siRNA on the expression of Arkadia, T $\beta$ RI, Smad7, SnoN and Ski in TGF- $\beta_{1}$-stimulated NHBECs. (a) Western blotting showing the effects of Arkadia siRNA, control siRNA and lactacystin on the protein expression of Arkadia, T $\beta$ RI, Smad7, SnoN and Ski in TGF- $\beta_{1}$-stimulated NHBECs. (b, c) The relative ratios of expression intensities of Arkadia, T $\beta$ RI, Smad7, SnoN and Ski to that of $\beta$-actin in TGF- $\beta_{1}$-stimulated NHBECs. (d) Real-time RT-PCR showing effects of Arkadia siRNA, control siRNA and lactacystin on the mRNA expression of Smad7, SnoN and Ski in TGF- $\beta_{1}$-stimulated NHBECs. Data are relative ratios of Smad7, SnoN and Ski mRNAs to $\beta$-actin mRNA. NHBECs, control NHBECs; TGF- $\beta_{1}$, NHBECs treated with TGF- $\beta_{1}$; Arkadia siRNA, TGF- $\beta_{1}$ stimulated NHBECs in the preincubation of Arkadia siRNA; control siRNA, TGF- $\beta_{1}$-stimulated NHBECs in the preincubation of control siRNA; and lactacystin, TGF- $\beta_{1}$-stimulated NHBECs in the preincubation of lactacystin. Experiments were repeated three times with similar results, and the data are expressed as the mean ratio \pm s.d. ${ }^{*} P<0.01$, compared with the control NHBECs; ${ }^{\mathbf{\Delta}} P<0.01$, compared with the TGF- $\beta_{1}$-stimulated NHBECs.

of T $\beta$ RI in TGF- $\beta_{1}$-stimulated NHBECs. In contrast to lactacystin, preincubation of Smurf 2 siRNA or control siRNA did not reverse the low expression of Smad7, SnoN and Ski proteins in TGF- $\beta_{1}$-stimulated NHBECs (Figure 6).

\section{DISCUSSION}

In this study, we showed that the lung of rats treated with OVA showed the progressive development of inflammatory infiltration and collagen deposition, which were associated 

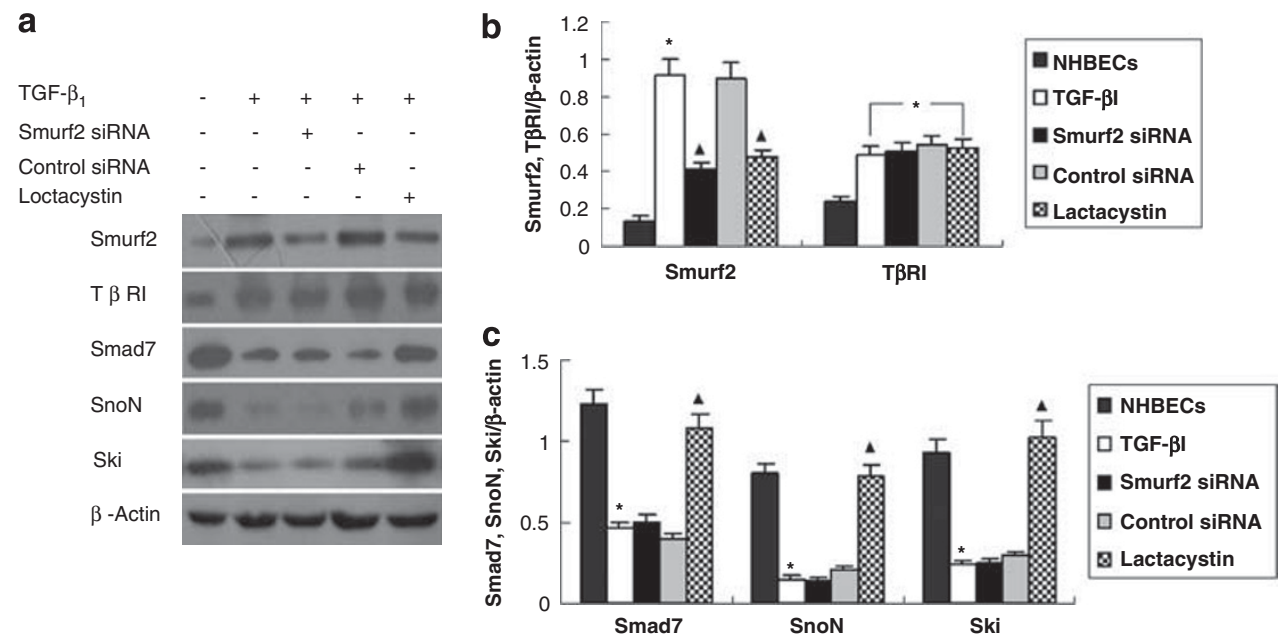

Figure 6 Effects of Smurf2 siRNA on the expression of Smurf2, T $\beta$ RI, Smad7, SnoN and Ski in NHBECs. (a) Western blotting showing effects of Smurf2 siRNA, control siRNA and lactacystin on the expression of Arkadia, T $\beta$ RI, Smad7, SnoN and Ski in TGF- $\beta_{1}$-stimulated NHBECs. (b, c) Relative ratios of expression intensities of Smurf2, T $\beta$ RI, Smad7, SnoN and Ski to that of $\beta$-actin in TGF- $\beta_{1}$-stimulated NHBECs. NHBECs, control NHBECs; TGF- $\beta_{1}$, TGF- $\beta_{1}$-stimulated NHBECs; Smurf2 siRNA, TGF- $\beta_{1}$-stimulated NHBECs in the preincubation of Smurf2 siRNA; control siRNA, TGF- $\beta_{1}$-stimulated NHBECs in the preincubation of control siRNA; and lactacystin, TGF- $\beta_{1}$-stimulated NHBECs in the preincubation of lactacystin. Experiments were repeated three times with similar results, and the data are expressed as the mean ratio \pm s.d. ${ }^{*} P<0.01$, compared with the control NHBECs; ${ }^{\mathbf{A}} P<0.01$, compared with the TGF- $\beta_{1}$-stimulated NHBECs.

with increased expression levels of TGF- $\beta_{1}$, COL-I, Smad7, SnoN and Ski mRNAs, but simultaneous diminished levels of Smad7, SnoN and Ski proteins. We then found that the expression of Arkadia and Smurf2, which are known to induce ubiquitin-dependent degradation of Smad7, SnoN and Ski, was increased in OVA-treated lungs. Finally, we used coimmunoprecipitation, which showed that Smurf2 interacted with Smad7, SnoN, Ski and T $\beta$ RI, and Arkadia only interacted with Smad7, SnoN and Ski, but not with T $\beta$ RI. These findings suggest that there is little possibility of decreased translation of Smad7, SnoN and Ski proteins, and that Arkadia and Smurf2 may contribute to the reduction of these three proteins in the pathogenesis of allergic airway remodeling.

Arkadia and Smurf2, two E3 ubiqutin ligases, have a pivotal role in regulating TGF- $\beta$ signaling through selectively targeting key components of the Smad pathway for degradation. ${ }^{11,12,20-23}$ Smurf2 is known to induce the degradation of TGF- $\beta$ receptors through interaction with Smad7, which enhances the inhibitory effect of Smad7 on TGF- $\beta$ signaling. ${ }^{19,22}$ In addition, Lin et $a l^{25}$ reported that Smurf2-regulated TGF- $\beta$ signaling by potently reducing the transcriptional activity of Smad2. However, Bonni et $a l^{23}$ have shown that Smurf2 may partner with phosphorylated Smad 2 to enhance TGF- $\beta$ signaling by degrading inhibitors such as SonN. When these findings are considered together, it seems that Smurf2 may exert bi-functional effects (ie, either amplify or inhibit) on the TGF- $\beta$ signaling through partnership with different classes of signal proteins and regulatory factors. As shown by immunoprecipitation, Smurf2 interacted with T $\beta R I$ in OVA-treated lungs. Previous studies have shown that Smurf2 induces the degradation of the T $\beta R I$ protein and enhances the inhibitory effect of negative reg- ulators on TGF- $\beta$ signaling. ${ }^{19,22}$ These results and our findings seem to indicate that Smurf2 could result in the inhibition of TGF- $\beta$ signaling in airway remodeling pathogenesis, which is contradictory to the notion that the upregulation of TGF- $\beta$ signaling is crucial for the pathogenesis of airway remodeling. There, we showed that T $\beta$ RI was increased in OVA-treated lungs. When these findings are considered together, it seems that Smurf2 is not competent to degrade the Smad7, SnoN and Ski in airway remodeling pathogenesis. Unlike Smurf2, Arkadia, which is widely expressed in embryonic and adult tissues, especially in lungs, is of relative specificity in modulation of TGF- $\beta$ signaling. Arkadia interacts strongly with Smad6/7, SnoN and Ski, degrades these proteins, ${ }^{20,21}$ and specifically activates the transcription of TGF- $\beta$ through Smad3/Smad4-binding sites and induces degradation of SnoN. ${ }^{26}$ In addition, it is very important that Arkadia is not recruited to TGF- $\beta$ receptors and does not degrade the receptors. ${ }^{20}$ In other words, Arkadia regulates the transcriptional activity of TGF- $\beta$ through degradation of Smad7, SnoN and Ski, but not TGF- $\beta$ receptors. Therefore, Arkadia amplifies TGF- $\beta$ signaling through degradation of Smad7, SnoN and Ski. ${ }^{20,21}$ Taken together, we presume that the reduction of Smad7, SnoN, and Ski could be mainly attributed to the high expression of Arkadia in allergic airway remodeling.

To confirm this presumption, we knocked down the expression of endogenous Arkadia and Smurf2 using siRNA in NHBECs. It was observed that there was a high level of T $\beta$ RI expression with low levels of Smad7, SnoN and Ski expression in TGF- $\beta_{1}$-stimulated NHBECs compared with control NHBECs. When NHBECs were preincubated with Arkadia siRNA and lactacystin, the reduction in the expression of Smad7, SnoN and Ski proteins induced by TGF- $\beta_{1}$ was partly 
reversed; however, the expression of $\mathrm{T} \beta \mathrm{RI}$ protein and Smad7/SnoN/Ski mRNA were not affected. In contrast to Arkadia siRNA, Smurf2 siRNA had no influence on the expression of Smad7, SnoN and Ski proteins. Similar to Arkadia siRNA and lactacystin, Smurf2 siRNA also did not affect the expression of $\mathrm{T} \beta \mathrm{R}$ I. In a previous study, Asano et $a l^{27}$ have shown that the overexpression of Smurf2 and coexpression of Smad7 along with Smurf2 all did not affect the levels of $\mathrm{T} \beta \mathrm{RI}$ protein in scleroderma fibroblasts. These findings, in agreement with ours, also suggest that Arkadiamediated positive regulation of TGF- $\beta$ signaling may have a promoting role in allergic airway remodeling.

In conclusion, Arkadia may contribute to the pathogenesis of airway remodeling through enhancing TGF- $\beta$ signaling by inducing the reduction of the expression of Smad7, SnoN and Ski proteins in OVA-sensitized and -challenged rats. Therefore, the identification of key molecular players such as Arkadia would be useful for therapeutic intervention in airway remodeling, and further investigation is required to determine the exact mechanisms.

\section{ACKNOWLEDGEMENT}

This work was partly supported by the China Postdoctoral Science Foundation (Grant No. 20080440994) and State Key Development of Basic Research of China (973 Program, Grant No. 2009CB522104).

\section{DISCLOSURE/CONFLICT OF INTEREST}

The authors declare no conflict of interest.

1. Broide DH. Immunologic and inflammatory mechanisms that drive asthma progression to remodeling. J Allergy Clin Immunol 2008;121:560-570.

2. Boxall C, Holgate ST, Davies DE. The contribution of transforming growth factor- $\beta$ and epidermal growth factor signalling to airway remodeling in chronic asthma. Eur Respir J 2006;27:208-229.

3. Liu X, Sun Y, Weinberg RA, et al. Ski/Sno and TGF- $\beta$ signaling. Cytokine Growth Factor Rev 2001;12:1-8.

4. Derynck R, Zhang Y, Feng XH. Smads: transcriptional activators of TGF$\beta$ responses. Cell 1998;95:737-740.

5. Dong C, Zhu S, Wang T, et al. Deficient Smad7 expression: a putative molecular defect in scleroderma. Proc Natl Acad Sci USA 2002;99:3908-3913.

6. Fukasawa $\mathrm{H}$, Yamamoto $\mathrm{T}$, Togawa $\mathrm{A}$, et al. Down-regulation of smad7 expression by ubiquitin-dependent degradation contributes to renal fibrosis in obstructive nephropathy in mice. Proc Natl Acad Sci USA 2004;101:8687-8692.

7. Liu FY, Li XZ, Peng YM, et al. Arkadia-Smad7-mediated positive regulation of TGF- $\beta$ signaling in a rat model of tubulointerstitial fibrosis. Am J Nephrol 2007;27:176-183.

8. Gonzalez AV, Le Bellego F, Ludwig MS. Imbalance of receptorregulated and inhibitory Smads in lung fibroblasts from bleomycinexposed rats. Am J Respir Cell Mol Biol 2007;36:206-212.
9. Venkatesan N, Pini L, Ludwig MS. Changes in Smad expression and subcellular localization in bleomycin-induced pulmonary fibrosis. Am J Physiol Lung Cell Mol Physiol 2004;287:L1342-L1347.

10. Wang B, Hao J, Jones SC, et al. Decreased Smad7 expression contributes to cardiac fibrosis in the infarcted rat heart. Am J Physiol Heart Circ Physiol 2002;282:H1685-H1696.

11. Tan $\mathrm{R}, \mathrm{He} \mathrm{W}$, Lin $\mathrm{X}$, et al. Smad ubiquitination regulatory factor-2 in the fibrotic kidney: regulation, target specificity, and functional implication. Am J Physiol Renal Physiol 2008;294:F1076-F1083.

12. Tan R, Zhang J, Tan X, et al. Downregulation of SnoN expression in obstructive nephropathy is mediated by an enhanced ubiquitin-dependent degradation. J Am Soc Nephrol 2006;17: 2781-2791.

13. Fukasawa $\mathrm{H}$, Yamamoto $T$, Togawa $A$, et al. Ubiquitin-dependent degradation of SnoN and Ski is increased in renal fibrosis induced by obstructive injury. Kidney Int 2006;69:1733-1740.

14. Yang J, Zhang X, Li Y, et al. Downregulation of Smad transcriptional corepressors SnoN and Ski in the fibrotic kidney: an amplification mechanism for TGF- $\beta_{1}$ signaling. J Am Soc Nephrol 2003;14: 3167-3177.

15. Nakao A, Fujii M, Matsumura $R$, et al. Transient gene transfer and expression of Smad7 prevents bleomycin-induced lung fibrosis in mice. J Clin Invest 1999;104:5-11.

16. $\mathrm{Li} \mathrm{JH}$, Zhu HJ, Huang XR, et al. Smad7 inhibits fibrotic effect of TGF- $\beta$ on renal tubular epithelial cells by blocking Smad2 activation. J Am Soc Nephrol 2002;13:1464-1472.

17. Lan HY, Mu W, Tomita N, et al. Inhibition of renal fibrosis by gene transfer of inducible Smad7 using ultrasound-microbubble system in rat UUO model. J Am Soc Nephrol 2003;14:1535-1548.

18. Nakao A, Sagara $\mathrm{H}$, Setoguchi $Y$, et al. Expression of Smad7 in bronchial epithelial cells is inversely correlated to basement membrane thickness and airway hyperresponsiveness in patients with asthma. J Allergy Clin Immunol 2002;110:873-878.

19. Ebisawa T, Fukuchi M, Murakami G, et al. Smurf1 interacts with transforming growth factor- $\beta$ type I receptor through Smad7 and induces receptor degradation. J Biol Chem 2001;276:12477-12480.

20. Koinuma $\mathrm{D}$, Shinozaki $\mathbf{M}$, Komuro $\mathrm{A}$, et al. Arkadia amplifies TGF- $\beta$ superfamily signalling through degradation of Smad7. EMBO J 2003;22:6458-6470.

21. Nagano $\mathrm{Y}$, Mavrakis $\mathrm{KJ}$, Lee $\mathrm{KL}$, et al. Arkadia induces degradation of SnoN and c-Ski to enhance transforming growth factor- $\beta$ signaling. J Biol Chem 2007;282:20492-20501.

22. Kavsak P, Rasmussen RK, Causing CG, et al. Smad7 binds to Smurf2 to form an E3 ubiquitin ligase that targets the TGF- $\beta$ receptor for degradation. Mol Cell 2000;6:1365-1375.

23. Bonni S, Wang HR, Causing CG, et al. TGF- $\beta$ induces assembly of a Smad2-Smurf2 ubiquitin ligase complex that targets SnoN for degradation. Nat Cell Biol 2001;3:587-595.

24. Liu FY, Li XZ, Peng YM, et al. Arkadia regulates TGF- $\beta$ signaling during renal tubular epithelial to mesenchymal cell transition. Kidney Int 2008;73:588-594.

25. Lin $X$, Liang $M$, Feng $X H$. Smurf2 is a ubiquitin E3 ligase mediating proteasome-dependent degradation of Smad 2 in transforming growth factor- $\beta$ signaling. J Biol Chem 2000;275:36818-36822.

26. Levy L, Howell M, Das D, et al. Arkadia activates Smad3/Smad4dependent transcription by triggering signal-induced $\mathrm{SnoN}$ degradation. Mol Cell Biol 2007;27:6068-6083.

27. Asano $\mathrm{Y}$, Ihn H, Yamane K, et al. Impaired Smad7-Smurf-mediated negative regulation of TGF- $\beta$ signaling in scleroderma fibroblasts. J Clin Invest 2004;113:253-264. 